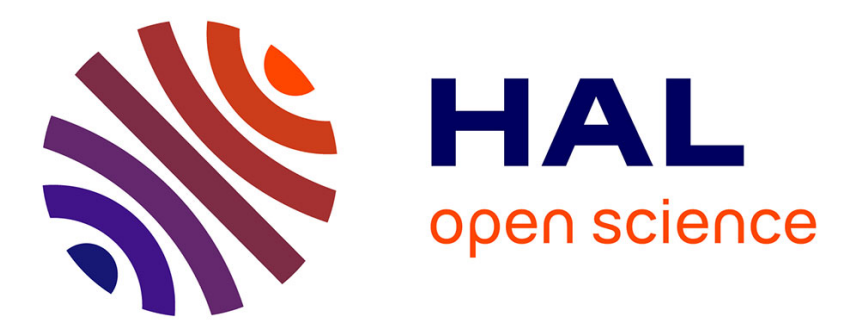

\title{
Évolution des emplois des marqueurs de topiques de discours dans Le Figaro de la fin du XIXe et du début du XXIe siècles
}

\author{
Michel Charolles, Sascha Diwersy, Denis Vigier
}

\section{- To cite this version:}

Michel Charolles, Sascha Diwersy, Denis Vigier. Évolution des emplois des marqueurs de topiques de discours dans Le Figaro de la fin du XIXe et du début du XXIe siècles. Langages, 2017, Du quantitatif au qualitatif en diachronie: prépositions françaises, 206, pp.85-104. 10.3917/lang.206.0085 . hal01907073

\section{HAL Id: hal-01907073 \\ https://hal.science/hal-01907073}

Submitted on 21 Apr 2020

HAL is a multi-disciplinary open access archive for the deposit and dissemination of scientific research documents, whether they are published or not. The documents may come from teaching and research institutions in France or abroad, or from public or private research centers.
L'archive ouverte pluridisciplinaire HAL, est destinée au dépôt et à la diffusion de documents scientifiques de niveau recherche, publiés ou non, émanant des établissements d'enseignement et de recherche français ou étrangers, des laboratoires publics ou privés. 


\title{
Evolution des emplois des marqueurs de topiques de discours dans Le Figaro de la fin du XIX et du début du XXI ${ }^{\mathrm{e}}$ siècles
}

\begin{abstract}
Evolution of the use of topic of discourse markers in Le Figaro of the end of the $\mathrm{XIX}^{\text {th }}$ and of the beginning of the $\mathrm{XXI}^{\text {st }}$ century
\end{abstract}

\author{
Michel Charolles \\ Université de Paris III \\ UMR-CNRS LATTICE ENS Paris
}

\author{
Sascha Diwersy \\ Université Paul Valéry Montpellier 3 \\ UMR-CNRS PRAXILING
}

Denis Vigier

Université Lumière Lyon 2

UMR-CNRS ICAR

\section{Résumé}

L'article porte sur l'usage des expressions à propos de, en matière (de), au sujet de, sur le plan (de), au niveau (de), dans le domaine (de), dans le secteur (de) et quant à comme marqueurs de topique de discours. Ces locutions présentent des différences aux niveaux morphosyntaxique et sémantique qui suggèrent qu'elles ne sont pas toutes grammaticalisées au même degré. C'est cette hypothèse que nous testons sur leurs emplois dans Le Figaro de la fin du $\mathrm{XIX}^{\mathrm{e}}$ siècle et du début du XXI siècle. La comparaison et l'étude statistique de leurs évolutions fréquentielles montre que ces expressions peuvent être réparties dans des sous-ensembles distincts de marqueurs de topique de discours, qui connaissent des tendances d'enracinement divergentes.

Mots clés : Marqueurs de topique de discours, grammaticalisation, lexicométrie

\begin{abstract}
The present paper is concerned with the use of the prepositional phrases à propos de, en matière (de), au sujet de, sur le plan (de), au niveau (de), dans le domaine (de), dans le secteur (de) and quant $\grave{a}$ as markers of topics in ongoing discourse. The differences which hold between these expressions at the syntactic, semantic and discourse level suggest that they are not grammaticalized to the same extent. This hypothesis is tested against two samples of newspaper texts from the end of the $19^{\text {th }}$ and the beginning of the $21^{\text {st }}$ century. Our analysis, which is underpinned by the results of frequency comparison, shows that the expressions under investigation form distinct sets of markers, which are involved in divergent trends of entrenchment.
\end{abstract}

Key words: discourse topic markers, grammaticalization, lexicometrics

\section{Introduction}

L'article qui suit porte sur une sélection de marqueurs pragmatiques du français du type de au sujet de, pour ce qui est de, quant à, à propos de, au niveau (de)...., qui ont pour fonction de guider les 
auditeurs/lecteurs dans la mise à jour des topiques des discours ${ }^{1}$. Les appellations utilisées pour désigner ces marqueurs varient d'un auteur à l'autre : Porhiel $(1998,2003)$ parle d'indicateurs d'intérêt, Charolles (1997) et Porhiel (2004 et 2005) d'introducteurs de cadres thématiques, Anscombre (2006), Velghe (2015), ainsi que Velghe \& Lahousse (2015) de marqueurs de thématisation. Dans la suite nous parlerons de marqueurs de topiques de discours, ce qui incite à les rapprocher des topiques de phrase dont ils diffèrent cependant (cf. Reinhart (1980 et 1981) et Van Dijk (1977) pour des études princeps).

Sans entrer dans les détails, nous considérerons que, si l'on peut en général sans difficultés affecter aux phrases (au moins catégoriques) un topique dont le prédicat dit quelque chose, il n'en va pas de même au niveau du discours (cf. Marandin, 1988, pour une étude pionnière). Dans le prolongement de Kuppevelt (1995), nous admettrons que dans une suite de deux phrases (P1 et P2), P1 n'a aucun topique de discours. P2 apporte un contenu dont on s'attend à ce qu'il réponde à une question que l'on s'est posée ou que l'on aurait pu se poser à la suite de $\mathrm{P} 1^{2}$. Défini de cette façon, le topique de discours local $^{3}$ correspond à l'orientation que prend le discours à chacune de ses avancées. Pour rendre compte de ces avancées, on a besoin de notions comme celles d'introduction, de continuation, de changement de topique de discours et de digression, qui correspondent à des enchaînements particuliers (sur ces notions cf. notamment Kuppevelt, 1995 ; Goutsos, 1996 ; Fraser, 2009a et 2009b).

Dans la première partie nous présentons la liste des marqueurs sur laquelle nous avons travaillé. En nous appuyant sur différents traits qui peuvent rapprocher ou différencier ces marqueurs aux niveaux morphosyntaxique et sémantique, nous avançons une hypothèse pour l'étude de leur grammaticalisation ${ }^{4}$, aspect qui est au centre de notre travail. Dans la deuxième partie nous présentons les résultats de l'annotation de ces marqueurs sur un échantillon de huit cents occurrences, prélevé aléatoirement sur l'un des corpus " complémentaires » de Presto (voir Diwersy, Falaise, Lay \& Souvay dans ce numéro). Cet échantillon réunit des numéros du quotidien Le Figaro de la fin du $\mathrm{XIX}^{\mathrm{e}}$ et du début du $\mathrm{XXI}^{\mathrm{e}}$ siècle et compte presque 70 millions de mots. Dans la troisième partie nous rapportons les résultats d'un calcul des spécificités (Lafon, 1980; Lebart \& Salem, 1994) mettant en évidence, pour chacune des expressions qui nous intéressent, des différences fréquentielles (en termes de sur-emploi / sous-emploi) entre les échantillons comparés. Dans la conclusion, nous confrontons nos résultats avec ceux présentés dans les analyses (non diachroniques) parues sur les marqueurs étudiés dans cet article et nous envisageons quelques pistes pour aller plus loin sur le sujet.

\section{Quelques marqueurs de topique de discours et hypothèses sur leur formation et leur évolution}

Les données sur corpus que nous avons rassemblées pour cette étude portent sur les expressions suivantes :

\footnotetext{
${ }^{1}$ Stricto sensu, il convient de distinguer les expressions plus ou moins figées comme à propos de, en matière (de), ... des Marqueurs de Topique de Discours proprement dits qui sont des SP : à propos de Paul, en matière linguistique,... et qu'il faudrait noter à propos de $S N / S A d j$ etc. Pour éviter le recours à des formulations fastidieuses, nous confondrons à dessein la notation de l'expression avec celle du Marqueur, sauf distinguo nécessaire dans l'argumentation.

${ }^{2}$ Sur ces questions dites « Question Under Discussion » (QUD), cf. notamment Roberts (2004).

${ }^{3}$ Ce topique de discours local ne correspond pas au topique de discours plus global (macrostructurel) de Van Dijk (1977).

${ }^{4}$ Par grammaticalisation on entend l'évolution linguistique qui soit change des lexèmes pleins en mots grammaticaux, soit rend des éléments grammaticaux encore plus grammaticaux. Depuis les travaux de Traugott (Traugott, 1989, 1995 ; Traugott \& König, 1991 ; Traugott \& Dasher 2002), la notion de grammaticalisation est proche de la pragmaticalisation (Lamiroy \& Charolles 2005): les mots passant à un sens plus abstrait, renvoient moins au monde concret (p.ex. relations spatiales) qu'à l'organisation de celui-ci par les locuteurs (p.ex. connecteurs et marqueurs de discours) et à leurs croyances (p.ex. modaux épistémiques).
} 
Toutes ces expressions se prêtent, en français contemporain, à des emplois comme marqueurs de topique de discours illustrés par les exemples suivants (tirés du corpus Le Figaro). Le critère sur lequel nous nous sommes fondés pour ranger ces emplois parmi les marqueurs de topique de discours est le suivant : l'expression (en gras) peut commuter ${ }^{5}$ avec les syntagmes $(e n+$ pour) ce qui concerne, concernant.

(1) Le président palestinien implore l'Union européenne de prendre le relais des Etats-Unis pour relancer la négociation. (...) A propos du cargo chargé d'armes arraisonné en mer Rouge, Arafat s'élève contre la version israélienne selon laquelle le chargement était destiné à l'Autorité palestinienne.

(2) Bernard Thibault réclame une sortie par le haut du système des multiples smic. Il faut, explique-t-il, arrêter de penser que le 'coût' social est la seule et unique variable d'ajustement de toute politique économique. En matière de retraite, il propose de cumuler les droits dès l'âge de 18 ans.

(3) La grande vision hyperbolique à la manière de Virgile et de Dante n'est pas son domaine. A ce sujet, le quatrième volume contient un texte étrange, énigmatique à la date du 8 août 1988.

(4) Les marchés anticipent ensuite une période de prudence tant qu'une image claire ne se dégagera pas des résultats et prévisions des sociétés. Sur le plan macro-économique, les nouvelles plus rassurantes sur le front du chômage et de la consommation devraient soutenir l'économie.

(5) [Il] assure que les programmes européens essayent d'encourager la distribution et la promotion des films. Au niveau de l'exploitation, une aide pour toutes les salles diffusant des films européens est demandée par les professionnels.

(6) Les ministres du Tourisme successifs ont répandu l'idée que la France était la première destination mondiale (...). Pourtant, le chiffre d'affaires ne suit pas. Dans ce domaine, la France n'est que troisième derrière les Etats-Unis et l'Espagne.

(7) Dans l'automobile, sa préférence va à Volkswagen (...) Dans le secteur bancaire, il cite UBS en raison de la solidité de son 'private banking' (banque privée) (...)

(8) Le classique triptyque 'informer, éduquer, divertir' ne suffit plus à identifier clairement la télévision publique. Si l'on en croit les mesures d'audience, les téléspectateurs français font plus confiance à la télévision commerciale lorsqu'ils souhaitent être informés ou divertis. Quant à la mission éducative, n'est-elle pas autant assurée par certaines chaînes thématiques privées que par celles créées dans le giron du secteur public?

Les locutions prépositionnelles regroupées supra sous (i) présentent des différences syntaxiques, morphologiques et sémantico-référentielles qui autorisent à les regrouper en sous-classes.

Parmi les expressions où figure un $\mathrm{N}$, seules celles construites avec plan, niveau, domaine, secteur, matière présentent une alternance de figement sur le modèle : (i) Prép. $(+$ Dét $)+N$ + de (suivi d'un SN); (ii) Prép. + (Dét.) + N (suivi d'un SAdj)

Tableau 1

Alternance de figement pour les locutions construites avec les $\mathrm{N}$ plan, niveau, domaine, secteur, matière

\begin{tabular}{|c|c|c|}
\hline $\begin{array}{c}\text { Nom figurant dans } \\
\text { la Prép / Loc Prép }\end{array}$ & LOC 1 & LOC 2 \\
\hline Propos $.(+$ Dét. $)+N+$ de & Prép. $(+$ Dét. $)+N$ \\
\hline
\end{tabular}

\footnotetext{
${ }^{5}$ Moyennant quelques ajustements morphosyntaxiques liés aux contraintes d'emploi de l'expression substituée.
} 


\begin{tabular}{|c|c|c|}
\hline matière & en matière de [stratégie] & en matière [stratégique] \\
\hline sujet & au sujet de [la stratégie] & *au sujet [stratégique] \\
\hline plan & sur le plan de [la stratégie] & sur le plan [stratégique] \\
\hline niveau & au niveau de [la stratégie] & au niveau [stratégique] \\
\hline domaine & dans le domaine de [la & dans le domaine \\
& [stratégie] & dane] \\
\hline secteur & dans lecteur de [la \\
& stratégie] & [stratégique] \\
\hline
\end{tabular}

Syntaxiquement, ces expressions présentent des différences constructionnelles. Si l'on choisit pour critère la catégorie morphosyntaxique de la tête de leur régime, on aboutit à un regroupement en deux grandes classes, la seconde pouvant elle-même être raffinée en deux sous-classes. Dans les tableaux suivants, nous proposons d'ajouter les expressions concernant, pour ce qui concerne, en ce qui concerne, et pour ce qui est de, afin d'équilibrer la comparaison entre les deux classes de marqueurs. Elles ne seront pas prises en compte dans l'étude sur corpus, qui ne portera que sur les marqueurs figurant dans la liste (i) supra.

Tableau 2

Premier classement des marqueurs sur le critère syntaxique de leur complémentation

\begin{tabular}{|c|c|c|c|c|c|c|}
\hline & \multirow{2}{*}{\multicolumn{2}{|c|}{ Préposition / locution prépositionnelle }} & \multicolumn{4}{|c|}{ Régime } \\
\hline & & & $\mathrm{SN}$ & $\mathrm{Npr}^{6}$ & ADJ & Vinf \\
\hline \multirow[t]{10}{*}{1} & \multirow[t]{2}{*}{ Sur le plan } & $d e$ & + & - & - & - \\
\hline & & $\varnothing$ & - & - & + & - \\
\hline & \multirow[t]{2}{*}{ Au niveau } & $d e$ & + & - & - & - \\
\hline & & $\varnothing$ & - & - & + & - \\
\hline & \multirow[t]{2}{*}{ Dans le domaine } & $d e$ & + & - & - & - \\
\hline & & $\varnothing$ & - & - & + & - \\
\hline & \multirow[t]{2}{*}{ Dans le secteur } & $d e$ & + & - & - & - \\
\hline & & $\varnothing$ & - & - & + & - \\
\hline & \multirow[t]{2}{*}{ En matière } & $d e$ & + & - & - & - \\
\hline & & $\varnothing$ & - & - & + & - \\
\hline \multirow[t]{5}{*}{$2 \mathrm{a}$} & & + & + & - & - \\
\hline & \multicolumn{2}{|l|}{ A propos de } & + & + & - & - \\
\hline & \multicolumn{2}{|l|}{ Concernant } & + & + & - & - \\
\hline & \multicolumn{2}{|l|}{ Pour ce qui concerne } & + & + & - & - \\
\hline & \multicolumn{2}{|l|}{ En ce qui concerne } & + & + & - & - \\
\hline \multirow[t]{2}{*}{$2 b$} & \multirow{2}{*}{\multicolumn{2}{|c|}{$\begin{array}{l}\text { Quant à } \\
\text { Pour ce qui est de }\end{array}$}} & + & + & - & + \\
\hline & & & + & + & - & + \\
\hline
\end{tabular}

On peut raffiner ce classement en introduisant un critère de variabilité morphosyntaxique ayant une contrepartie sémantico-référentielle: l'expression présente-t-elle ou non une variabilité lui permettant d'inclure une reprise anaphorique?

Tableau 3

Possibilité pour certains marqueurs d'inclure une reprise anaphorique

\begin{tabular}{|c|c|}
\hline Prép / Loc Prép & Reprise anaphorique \\
\hline sur le plan (de) & sur ce plan \\
\hline au niveau (de) & à ce niveau \\
\hline
\end{tabular}

\footnotetext{
${ }^{6}$ Nom propre.
} 


\begin{tabular}{|c|c|}
\hline dans le domaine $(d e)$ & dans ce domaine \\
\hline dans le secteur $($ de) & dans ce secteur \\
\hline en matière $($ de) & en (cette + la) matière \\
\hline au sujet de & à ce sujet \\
\hline à propos de & à ce propos \\
\hline concernant & $\varnothing$ \\
\hline pour ce qui concerne & $\varnothing$ \\
\hline quant à & $\varnothing$ \\
\hline pour ce qui est de & $\varnothing$ \\
\hline
\end{tabular}

On aboutit finalement au regroupement suivant :

Tableau 4

Regroupement en quatre sous-classes des expressions prises en compte dans les tableaux 2 et 3

\begin{tabular}{|c|c|c|c|}
\hline $\begin{array}{c}\text { Sur le plan (de) } \\
\text { Au niveau (de) } \\
\text { Dans le domaine (de) } \\
\text { Dans le secteur (de) }\end{array}$ & & $\begin{array}{c}\text { Concernant } \\
\text { En ce qui concerne } \\
\text { Pour ce qui concerne }\end{array}$ & \\
\hline En matiere (de) & $\begin{array}{l}\text { A propos de } \\
\text { Au sujet de }\end{array}$ & & $\begin{array}{c}\text { Quant à } \\
\text { Pour ce qui est de }\end{array}$ \\
\hline
\end{tabular}

Ce tableau donne une photographie de quelques-unes des formes à même de fonctionner en français contemporain comme des marqueurs de topique de discours. Ces formes ne sont visiblement pas toutes aussi spécialisées dans ce genre de fonction. Les expressions et locutions sans $\mathrm{N}$ comme quant à et pour ce qui est de que l'on a coutume - comme concernant, pour/en ce qui concerne d'appeler topicalisateurs (Combettes \& Prévost, 2001, 2003) semblent majoritairement, voire exclusivement, employées en français contemporain comme des marqueurs de topique de discours (Prévost, 2003, 2007 et 2010). Cela est assez évident avec quant à où l'on a du mal à imaginer aujourd'hui des usages comme *il m'a parlé quant à Paul, ?? Marie estime quant à Paul qu'il est très serviable. Il en va autrement avec sur le plan (de), au niveau (de), dans le domaine (de), dans le secteur (de) et en matière (de) où les emplois non idiomatiques viennent facilement à l'esprit : les scouts ont campé dans le domaine du château; il faut respecter le tracé sur le plan de l'architecte; la rivière est montée jusqu'au niveau du $1^{\text {er }}$ étage; ce mur est construit en matière végétale; il s'est implanté dans le secteur de $X$.

Si l'on se tourne vers les dictionnaires, on observe que les expressions appartenant à la seconde classe du tableau 2 sont plus souvent recensées comme locutions figées (sauf pour ce qui est de et en ce qui concerne) que celles du premier groupe. De même pour leur datation : les données fournies par le Trésor de la Langue Française informatisé (TLFi) et Le Dictionnaire Historique de la Langue Française (DHLF) aussi bien que celles que nous avons recueillies sur Frantext font apparaître une opposition entre d'un côté sur le plan (de), au niveau (de), dans le domaine (de), dans le secteur (de) qui sont de formation récente, et de l'autre quant à attesté dès l'ancien français ; avec, entre les deux, au sujet de, à propos de et en matière (de).

Tableau 5

Locutions figées recensées (ou non) dans le Trésor de la Langue Française informatisé (TLFi) et Le Dictionnaire Historique de la Langue Française (DHLF) Date de première attestation dans Frantext 


\begin{tabular}{|c|c|c|c|c|}
\hline & \multicolumn{2}{|c|}{$\begin{array}{l}\text { Expression notée } \\
\text { comme locution }\end{array}$} & \multicolumn{2}{|c|}{ Date de $1^{\text {ère }}$ attestation } \\
\hline & $T L F i$ & DHLF & $\begin{array}{c}\text { Dans } \\
T L F i / D H L F\end{array}$ & $\begin{array}{c}\text { dans Frantext } \\
(235000000 \text { mots })^{7}\end{array}$ \\
\hline Dans le domaine (de) & - & - & & 1874 (SN), 1923 (Adj) \\
\hline Sur le plan (de) & + & - & & 1934 (SN), 1925 (Adj) \\
\hline Au niveau (de) & - & + & $(1778)^{8}$ & 1942 (SN), 1948 (Adj) \\
\hline Dans le secteur (de) & - & - & & $1958(\mathrm{SN}), 1960$ (Adj) \\
\hline En matière (de) & - & + & & 1633 (SN), 1790 (Adj) \\
\hline Au sujet de & + & + & 1617 & 1734 (SN/Npr) \\
\hline A propos de & + & + & 1580 & 1552 (SN/Npr) \\
\hline Quant à & + & + & $1269 / 1278$ & 1347 (SN/Npr) \\
\hline Pour ce qui est de & - & - & & 1593 (SN/Npr) \\
\hline Concernant & + & + & & 1789 (SN/Npr) \\
\hline Pour ce qui concerne & + & + & $1690 / 1690$ & 1601 (SN/Npr) \\
\hline En ce qui concerne & + & + & $1690 / 1690$ & 1833 (SN/Npr) \\
\hline
\end{tabular}

Le fait que en matière (de) soit employé comme marqueur de topique depuis le $\mathrm{XVII}^{\mathrm{e}}$ et $\mathrm{XVIII}{ }^{\mathrm{e}}$ siècle, bien avant sur le plan (de), au niveau (de), dans le domaine (de), dans le secteur (de), incite à le disjoindre de ces marqueurs dans le tableau 4 et à le rapprocher de à propos de et de au sujet de dont les premières attestations datent de la même période. Ce rapprochement se justifie sémantiquement dans la mesure où le $\mathrm{N}$ matière est plus proche des $\mathrm{N}$ sujet et propos avec qui il peut partager l'idée de 'contenu verbal', alors qu'il n'a aucun rapport évident avec les $\mathrm{N}$ spatiaux plan, niveau, domaine, secteur. À ce propos, il n'est pas sans intérêt de relever que quant à, qui est le marqueur de topique de discours le plus spécialisé, le plus ancien et le plus étudié (Choï-Jonin, 1997 ; Fløttum, 1999, 2003 ; Debaisieux, 2001 ; Anscombre, 2006 ; Prévost, 2003a, 2008, 2010 ; Lagae, 2003), se différencie radicalement des autres marqueurs par son opacité sémantique, au point que l'on peut sans risque faire l'hypothèse qu'il est le plus grammaticalisé, au contraire de sur le plan de/Adj, au niveau de/Adj, dans le domaine de/Adj, dans le secteur de/Adj, qui semblent beaucoup moins avancés sur ce chemin.

Tableau 6

Hypothèse sur les positions relatives occupées par les expressions étudiées sur une échelle de grammaticalisation

\begin{tabular}{|l|c|c|c|}
\hline $\begin{array}{l}\text { Sur le plan (de) } \\
\text { Au niveau (de) } \\
\begin{array}{l}\text { Dans le domaine (de) } \\
\text { Dans le secteur (de) }\end{array}\end{array}$ & En matière (de) & $\begin{array}{c}\text { A propos de } \\
\text { Au sujet de }\end{array}$ & Quant à \\
\hline $\begin{array}{l}\text { - grammaticalisées } \\
\text { (formation) récente }\end{array}$ & $\longrightarrow$ & $\begin{array}{r}\text { + grammaticalisées } \\
\text { (formation) ancienne }\end{array}$ \\
\hline
\end{tabular}

\footnotetext{
${ }^{7}$ Interrogation sur chacune des expressions étudiées précédées d'un point, pour sélectionner leurs emplois en tête de phrase où elles ont plus de chances d'être des marqueurs de topique de discours.

${ }^{8}$ Après avoir noté que le $\mathrm{N}$ niveau a pris au XVIII ${ }^{\text {ème }}$ siècle un sens abstrait pour parler de la position sociale ou économique, le DHLF relève (p. 2376) que « les locutions au niveau de (av. 1778) suivent ces emplois abstraits. Elles sont devenues au $\mathrm{XX}^{\text {ème }}$ siècle des emplois passe-partout diffusés par le langage des ingénieurs, pour « dans le domaine de », «pour» et sont critiquées ». La datation «av. $1778 »$ ne vaut pas pour ces emplois contemporains qui nous intéressent.
} 


\section{Emplois des marqueurs de topique du discours dans Le Figaro de la fin du XIX et du début du XXI ${ }^{\mathrm{e}}$ siècle. Étude sur corpus.}

Pour tester l'hypothèse ci-dessus, nous proposons de nous tourner vers le corpus complémentaire de Presto qu'est le Corpus diachronique de la presse française, vaste échantillon de périodiques

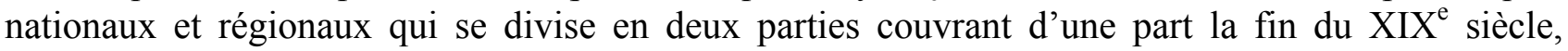
d'autre part le début du XXI ${ }^{\mathrm{e}}$ siècle. L'ensemble du corpus a été catégorisé et lemmatisé au moyen de l'analyseur TreeTagger (Stein \& Schmid 1995).

\subsection{Constitution du Corpus}

Pour constituer notre corpus de travail, nous avons sélectionné les articles du quotidien Le Figaro, ce qui nous donne deux sous-corpus, dont le tableau suivant présente un aperçu :

\begin{tabular}{|l|l|l|r|}
\hline Sous-corpus & Années & $\begin{array}{l}\text { Total } \\
\text { numéros }\end{array}$ & \multicolumn{1}{|l|}{ Total mots-occurrences } \\
\hline $\begin{array}{l}\text { Le Figaro XIX }{ }^{\text {ème }} \text { siècle } \\
{[\text { désormais } L F-19]}\end{array}$ & $1885,1890,1895,1896$ & 964 & 37.446 .988 \\
$\begin{array}{l}\text { Le Figaro XXI }{ }^{\mathrm{e}} \text { siècle } \\
\text { [désormais } L F-21]\end{array}$ & 2002 & 313 & 30.497 .189 \\
\hline TOTAUX & 1277 & 67.944 .177 \\
\hline
\end{tabular}

La plateforme web $\mathrm{BTLC}^{9}$ donne accès à différentes applications permettant d'explorer ce corpus ; ces applications sont implémentées par la boîte à outils PrimeStat dont les fonctionnalités suivantes ont été utilisées pour cette étude : création de sous-corpus et de partitions, définition d'expressions de requête complexes en termes de propriétés lexicales et de nombre de segments ciblés, extraction et manipulation de concordances KWIC, calcul de spécificités fréquentielles.

\subsection{Fréquence d'occurrence des marqueurs de topique du discours étudiés.}

Notre objectif consiste à nous faire une idée la plus précise possible de l'évolution des usages dans notre corpus des marqueurs :

à propos de, en matière (de), au sujet de, sur le plan (de), au niveau (de), dans le domaine (de), dans le secteur (de), quant à

Pour ce faire, nous avons comparé les fréquences relatives de ces expressions dans les deux souscorpus considérés comme deux tranches synchroniques.

Nous avons d'abord extrait au sein des deux sous-corpus LF-19 et LF-21 les expressions recherchées [étape E1]. Parmi les occurrences extraites figuraient des séquences non pertinentes pour notre recherche. Afin d'évaluer quelle part occupait le «bruit» dans cette première étape, nous avons constitué pour chaque expression étudiée un échantillon de cent occurrences ${ }^{10}$ tirées aléatoirement parmi celles obtenues à l'issue de E1. Soit huit cents occurrences triées et codées en tout [étape E2].

Notre codage, outre la catégorie « bruit », a distingué deux critères de classement :

\footnotetext{
${ }^{9}$ Plateforme développée (ainsi que PrimeStat) par S. Diwersy, d'abord à l'Université de Cologne, et depuis 2015 à l'Université de Montpellier 3.

${ }^{10} 50$ occurrences pour LF-19 et 50 pour LF-21
} 
(i) un critère sémantique : emploi topique [TOP] - non topique [Non TOP]

(ii) un critère syntaxique: position intraprédicative versus extraprédicative (Guimier $1996: 5)$

Le travail a été accompli par deux experts séparément et a été suivi d'une phase de discussion autour des occurrences dont le codage divergeait, jusqu'à accord des annotateurs.

Pour chaque marqueur ont été calculés les pourcentages recueillis par chacune des cinq souscatégories distinguées et codées au sein de chaque échantillon. Ces pourcentages ont permis de quantifier par extrapolation la fréquence attendue des occurrences relevant de chacune des cinq sous-catégories dans LF-19 et dans LF-21. Ces fréquences théoriques ont ensuite été pondérées en fréquences relatives. Les valeurs ainsi obtenues ont été multipliées par $10^{7}$ pour aboutir à des fréquences plus aisément interprétables et comparables [étape E3].

\subsection{Résultats}

Voici le diagramme des fréquences relatives calculées pour les catégories (sémantiques) TOP et Non-TOP après extrapolation des résultats obtenus à l'issue du codage des échantillons.

\section{Diagramme 1}

Diagramme des fréquences relatives (pour 10000000 ) des marqueurs pour les catégories sémantiques TOP et Non-TOP. Corpus LF-19 et LF-21

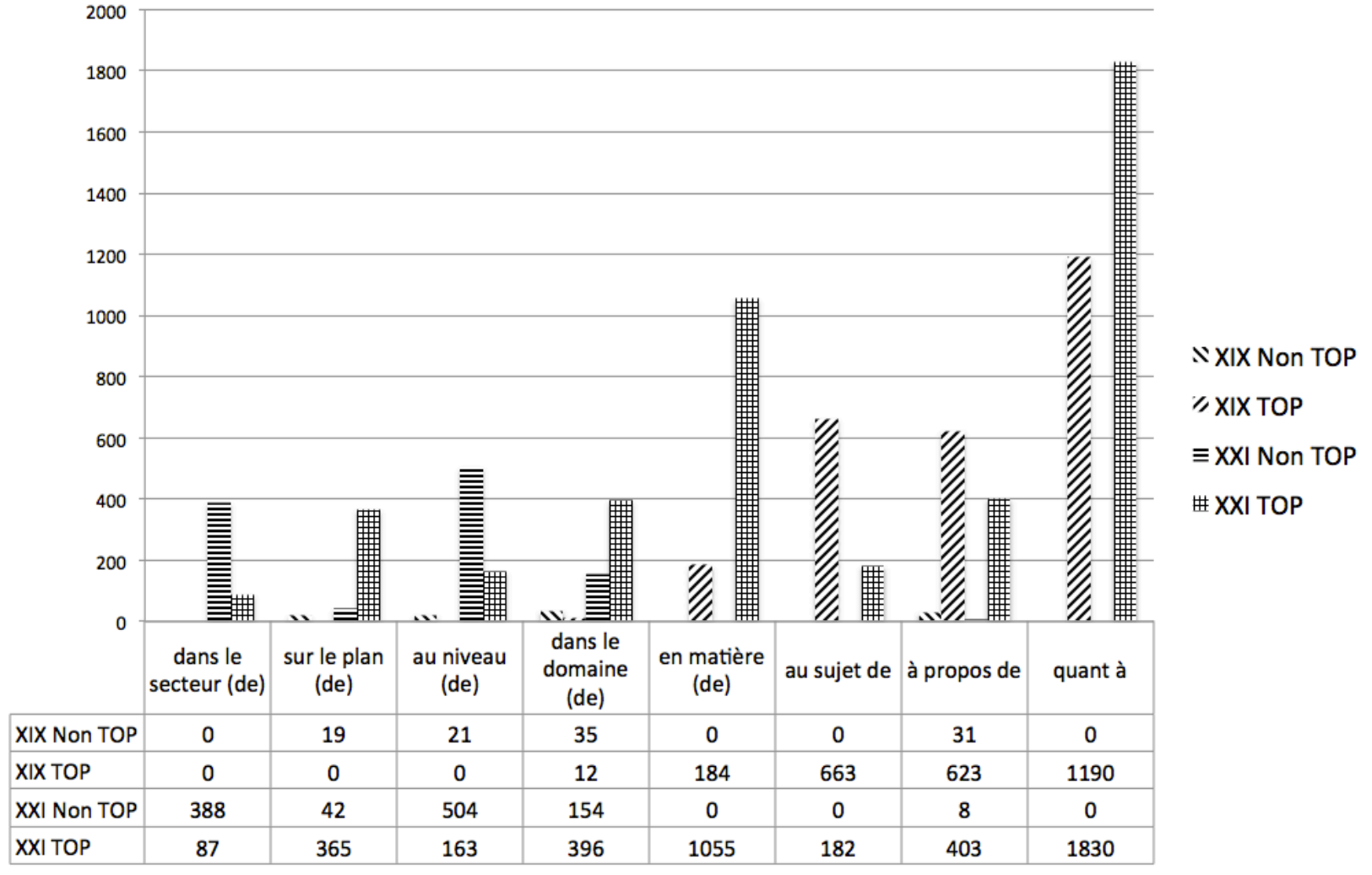

A la fin du XIX $\mathrm{X}^{\mathrm{e}}$ siècle, deux groupes de marqueurs se détachent nettement avec d'un côté, dans le secteur (de), sur le plan (de), au niveau (de), dans le domaine (de) dont l'usage est rare (voire inexistant) et presque exclusivement cantonné à une valeur non-topique ; de l'autre côté, en matière de, au sujet de, à propos de et quant à qui s'utilisent dès cette période comme marqueurs de topiques de discours - que ce soit exclusivement pour en matière (de), au sujet de, quant à, ou majoritairement pour à propos de.

$\mathrm{Au}$ début du XXI $\mathrm{X}^{\mathrm{e}}$ siècle, on observe plusieurs changements. Dans le secteur (de), sur le plan (de), au niveau (de) présentent dans LF-21 des emplois comme marqueurs de topique de discours, 
inexistants dans LF-19; quant à dans le domaine (de), il est déjà employé avec cette valeur TOP dans LF-19 mais avec une fréquence relative très inférieure à ce qu'elle est au $\mathrm{XXI}^{\mathrm{e}}$ siècle. Cette évolution est assez remarquable pour une si courte période et si l'on tient compte du fait qu'elle se traduit, pour les expressions en question, par un changement sémantique et fonctionnel important. Quant à reste de loin le plus topical des marqueurs : il présente plus d'emplois de ce type qu'au $\mathrm{XIX}^{\mathrm{e}}$ siècle et devance nettement au sujet de et à propos de dont les emplois comme topique de discours déclinent du $\mathrm{XIX}^{\mathrm{e}}$ au $\mathrm{XXI}^{\mathrm{e}}$ siècle. En matière (de) qui, comme on l'a relevé, ne connaît dès le $\mathrm{XIX}^{\mathrm{e}}$ siècle que des emplois comme marqueur de topique de discours, persiste dans cette exclusivité et son usage s'intensifie entre la fin du XIX ${ }^{\mathrm{e}}$ siècle et le début du XXI ${ }^{\mathrm{e}}$ siècle.

Voici un second diagramme qui présente les fréquences relatives des expressions étudiées, selon qu'elles occupent une position intraprédicative ou extraprédicative dans leurs emplois topicaux.

\section{Diagramme 2}

\section{Fréquences relatives (pour 10000000 ) des marqueurs pour les catégories TOP intraprédicatif} et TOP extraprédicatif. Corpus LF-19 et LF-21

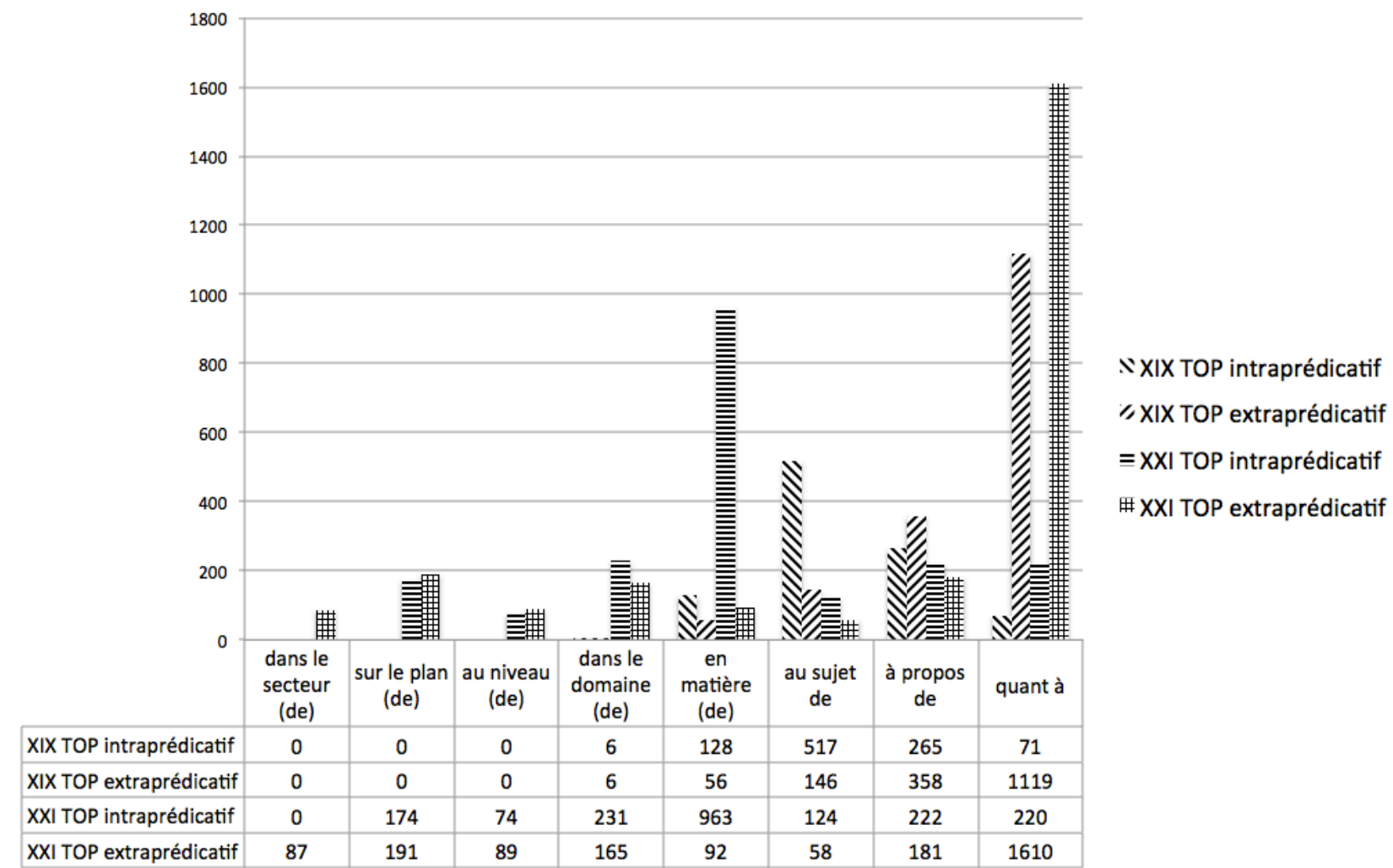

On retrouve le contraste entre dans le secteur (de), sur le plan (de), au niveau (de), dans le domaine (de) et les autres marqueurs, les premiers n'émergeant comme TOP que dans la seconde période. Les fréquences pour dans le secteur (de) et au niveau (de) sont particulièrement faibles, ce qui tend à les distinguer des deux autres qui, sous ce rapport, s'approchent des valeurs fréquentielles de $\grave{a}$ propos de.

Voici deux exemples d'emploi de dans le domaine (de) respectivement intraprédicatif (9) et extraprédicatif (10) :

(9) Dans nombre de cas, les journaux n'avaient-ils pas signalé l'ingérence quotidienne du président du Conseil dans le domaine des affaires extérieures, ses entretiens fréquents avec tels ou tels membres du corps diplomatique étranger? (LF-19)

(10) On est bien entendu très loin de l'explosive affaire Enron. Mais l'on sent bien que la crainte cachée de tous est qu'un équivalent ne se produise en France. Or dans le domaine de l'honnêteté, il n'y a pas de sécurité possible. (LF-21) 
Quant à présente beaucoup d'emplois extraprédicatifs (11) mais il garde des emplois intraprédicatifs à la fin du $\mathrm{XIX}^{\mathrm{e}}$ et au début du XXI $\mathrm{X}^{\mathrm{e}}$, comme (12), quoique dans une faible proportion :

(11) Le texte est de notre collaborateur M. Jules Richard que ses connaissances des choses militaires désignaient tout naturellement pour la partie littéraire. Quant à M. Détaille, il a résolu le problème de faire revivre les types de l'armée sans tomber dans la monotonie. (LF-19)

(12) La compétence 'teutonne' de notre historienne est à ce point considérable qu'on lui pardonne volontiers ces petites lacunes quant aux documents de la francité. Ajoutons que vichystologue, elle n'est pas nécessairement vichystophage, performance qui n'est pas si fréquente par le temps qui court. (LF-21)

Au sujet de et à propos de ont des profils assez différents : avec au sujet de, les emplois intraprédicatifs l'emportent sur les extraprédicatifs aux deux périodes. A propos de a nettement plus d'emplois extraprédicatifs que au sujet de, sur les deux périodes, mais ces emplois ne sont pas majoritaires au XXI ${ }^{\mathrm{e}}$ siècle. Le profil de en matière (de) est à la fois différent de celui de au sujet (de) et de à propos (de) mais aussi de quant à. En matière (de) présente en effet beaucoup plus d'emplois intraprédicatifs (comme (13)) qu'extraprédicatifs (comme (14)) à chaque période et cet avantage tend à s'accentuer au $\mathrm{XXI}^{\mathrm{e}}$ siècle :

(13) Après Denis Olivennes, le patron opérationnel de Canal + qui s'exprimait devant le Parti socialiste il y a deux mois, et Pierre Lescure qui, en décembre dernier, le martelait à qui voulait l'entendre, le voici qui réclame, à son tour, l' "équité" devant les obligations de production en matière de cinéma. (LF-21)

(14) Espérons qu'on reviendra sur cette chinoiserie. Mais que voulez-vous, en matière d'avancement, on individualise, on ne généralise pas. (LF-21)

Si en matière (de) et quant à ont nettement plus d'emplois comme TOP au XXI $\mathrm{e}^{\mathrm{e}}$ siècle que tous les autres, cette suprématie n'a pas la même origine : elle tient, avec quant à, à la prééminence des emplois extraprédicatifs et, avec en matière (de), à celle des emplois intraprédicatifs.

Le diagramme ci-dessous présente les résultats d'un test que nous avons appliqué aux extraits comportant sur le plan (de), au niveau (de), dans le domaine (de), dans le secteur (de) et en matière (de) TOP intra- et extraprédicatifs. Ce test vise à départager, parmi les emplois de ces marqueurs comme TOP, ceux qui semblent plus topicaux que d'autres. Le test utilisé ne s'applique pas aux occurrences de au sujet de et à propos de parce que tout simplement il repose sur la possibilité de commuter tel emploi de ces marqueurs soit avec à propos de / au sujet de (TOP ++$)$ soit avec concernant len/pour ce qui concerne $(\mathrm{TOP}+)^{11}$.

\section{Diagramme 3 \\ Pourcentages de marqueurs codés « TOP+» et « TOP++ » dans chacun des deux sous-corpus : LF-19 et LF-21}

\footnotetext{
${ }^{11}$ Le fait de considérer que les emplois dans lesquels sur le plan de/Adj, au niveau de/Adj, dans le domaine de/Adj, dans le secteur de/Adj et en matière de/Adj commutent avec à propos (de) et au sujet (de) sont les plus topicaux remonte à Reinhart (1980) et (1981) qui en fait un test pour identifier les topiques de discours, et elle est reprise par Velghe \& Lahousse (2015) et Velghe (2015). Sur à propos de voir Prévost (2007, 2011), Lagae (2011) et Porhiel (2001) qui le compare à au sujet de.
} 


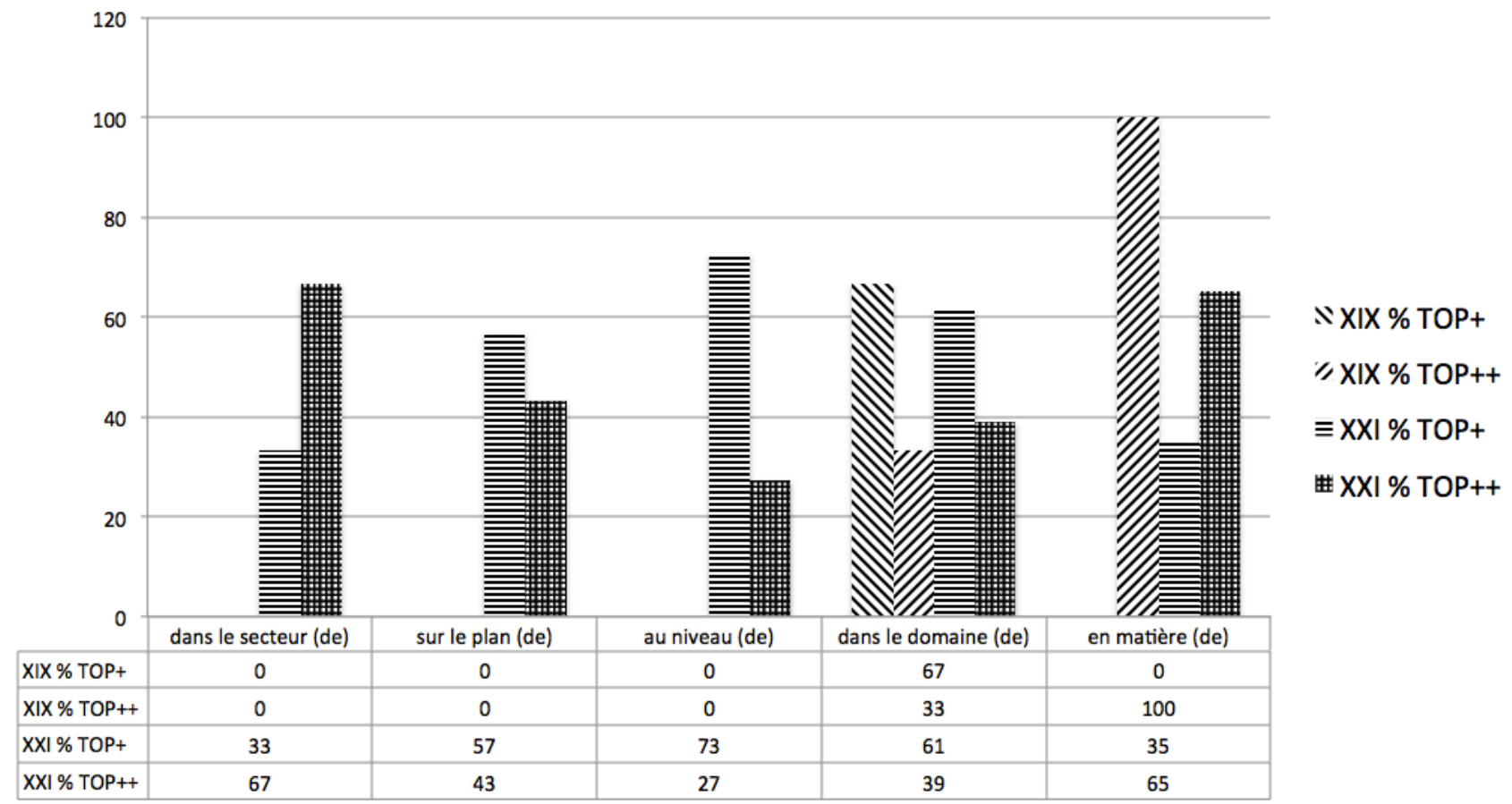

Là encore, en matière (de) se distingue des autres marqueurs puisque ses emplois les plus topicaux dominent dès le $\mathrm{XIX}^{\mathrm{e}}$ siècle. À l'inverse, dans le secteur (de), sur le plan (de), au niveau (de) voient leur emploi TOP++ n'apparaître qu'au XXI ${ }^{\mathrm{e}}$ siècle (au niveau (de) se classant en dernier). Enfin, dans le domaine (de) occupe une position intermédiaire : quoiqu'on le rencontre dans des emplois très topicaux dès le $\mathrm{XIX}^{\mathrm{e}}$ siècle, ces derniers s'avèrent minoritaires aux $\mathrm{XIX}^{\mathrm{e}}$ et $\mathrm{XXI}^{\mathrm{e}}$ siècles.

\section{Mise en œuvre de calculs de spécificités}

Pour compléter les analyses présentées dans la section précédente et qui s'appuient sur l'examen des fréquences relatives calculées pour les deux sous-corpus LF-19 et LF-21, nous recourrons ci-dessous au calcul des spécificités ${ }^{12}$ (cf. Lafon 1980). Dans un premier temps, nous avons fait un calcul de manière à déterminer si les marqueurs étudiés s'avéraient sur- ou sous-représentés dans chacune des deux parties (LF-19 et LF-21) de notre corpus total. Voici les indices auxquels nous parvenons :

\section{Diagramme 4}

Indices de spécificité calculés pour les marqueurs employés comme topiques quelle que soit leur position syntaxique (intraprédicatifs et extraprédicatifs). Corpus total partitionné en deux parties (LF-19 et LF-21)

\footnotetext{
${ }^{12}$ Pour une présentation de ce calcul et des paramètres qu'il met en jeu, voir D. Vigier dans ce numéro.
} 


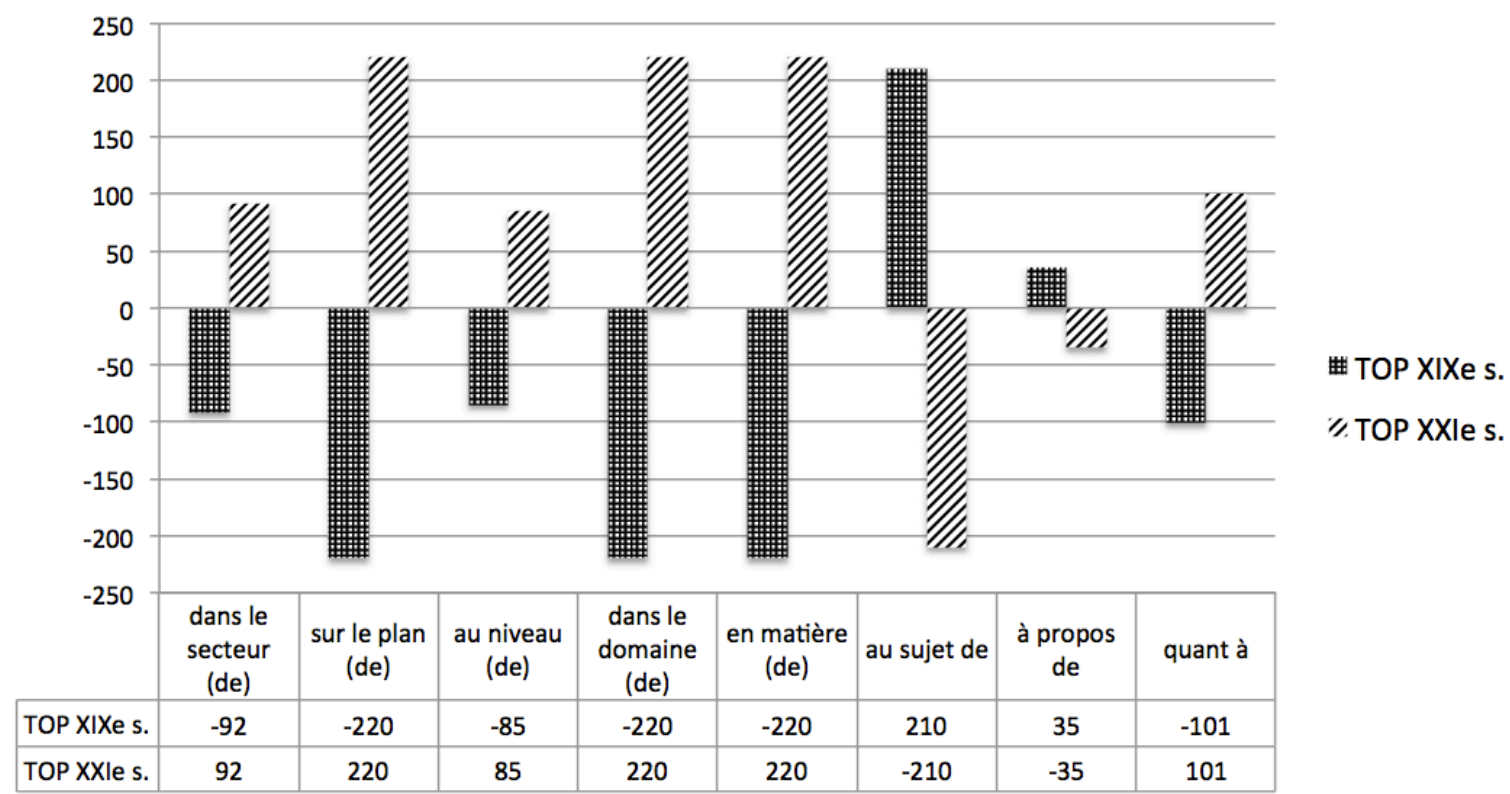

Ce qui frappe d'abord, c'est qu'aucun des marqueurs étudiés ne possède une distribution «banale» (au sens statistique) dans les deux parties du corpus ${ }^{13}$ : tous sont soit sur-représentés soit sous-représentés. Notre corpus nous invite donc à considérer que quel que soit le marqueur, il se passe quelque chose de statistiquement significatif quant à son usage comme marqueur TOP entre le $\mathrm{XIX}^{\mathrm{e}}$ et le $\mathrm{XXI}^{\mathrm{e}}$ siècle dans le journal Le Figaro.

En second lieu, il est frappant également que la répartition des sur- et des sous-emplois de au sujet de, à propos de dans les deux parties du corpus soit symétrique de celle observée pour les autres marqueurs. Tandis que les deux premiers sont sous-représentés au $\mathrm{XXI}^{\mathrm{e}}$ siècle, les autres s'avèrent sur-représentés pour la même période. On retrouve ici la désaffection relative qui frappe l'usage de ces deux marqueurs dans notre corpus et que la comparaison des fréquences relatives révélait déjà.

Autre observation : les «profils » de sur- et de sous-emploi des trois marqueurs sur le plan (de), dans le domaine (de) et en matière (de) apparaissent très proches dans le diagramme. Le calcul montre en effet que, pour ce qui regarde leur usage comme marqueurs de topique, ce sont eux qui connaissent les plus grands changements entre le $\mathrm{XIX}^{\mathrm{e}}$ et le $\mathrm{XXI}^{\mathrm{e}}$ siècle. Le sort de dans le secteur (de) et de au niveau (de) - aux profils proches aussi - est à cet égard moins contrasté. Et c'est à propos de qui présente finalement le moins de contraste en termes d'usage comme TOP entre les deux périodes considérées.

Faut-il enfin s'étonner que les indices de sur- et de sous-spécificité affectés à quant à soient très inférieurs à ceux calculés pour sur le plan (de), dans le domaine (de) et en matière (de) notamment, alors que nous avons plusieurs fois souligné sa très haute fréquence d'emploi comme topique aux $\mathrm{XIX}^{\mathrm{e}}$ et $\mathrm{XXI}^{\mathrm{e}}$ siècles ? En fait, il n'y a là rien d'étonnant : les deux indices calculés montrent en effet que si quant à est (encore) plus souvent utilisé comme TOP dans Le Figaro $\mathrm{XXI}^{\mathrm{e}}$ siècle qu'il ne l'était dans Le Figaro $\mathrm{XIX}^{\mathrm{e}}$ siècle, le contraste entre sa fréquence d'emploi comme TOP entre ces deux périodes est statistiquement moins significatif qu'il ne l'est pour les trois autres marqueurs.

Alors que le diagramme 4 présentait les indices de spécificité calculés pour les emplois TOP des marqueurs étudiés dans les deux parties de notre corpus quelle que soit leur position syntaxique, le diagramme 5 restreint ce calcul à leurs seuls emplois TOP extraprédicatifs.

\footnotetext{
${ }^{13}$ ce qui serait le cas si la valeur absolue de l'indice de spécificité était inférieure (au sens strict) à 3 .
} 


\section{Diagramme 5}

Indices de spécificité calculés pour les marqueurs employés comme topiques extraprédicatifs. Corpus total partitionné en deux parties (LF-19 et LF-21)

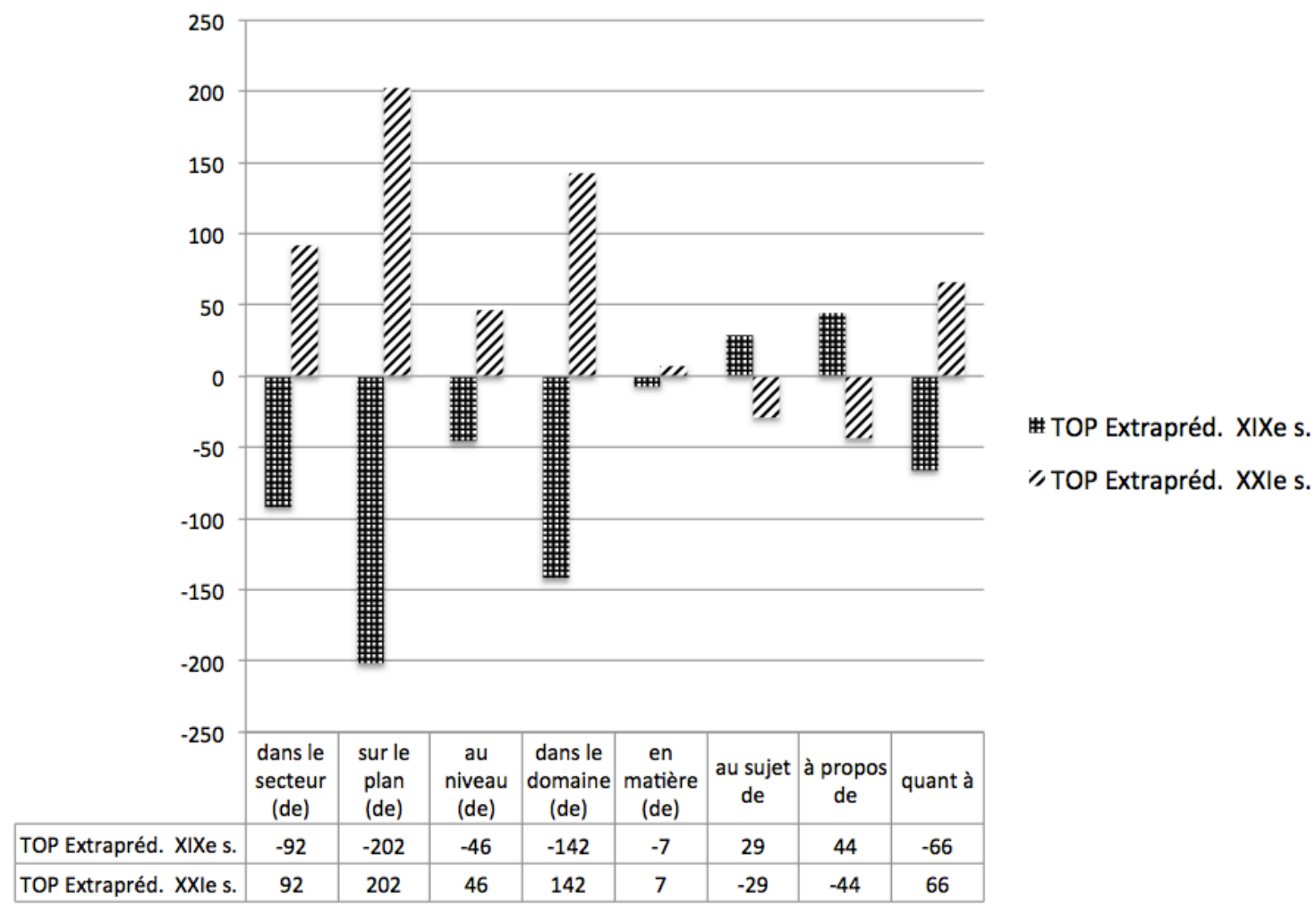

On observera d'abord la relative faiblesse des deux indices affectés aux fréquences d'emplois de en matière (de). Si on les compare à celui affecté au même marqueur dans le diagramme 4, on est frappé par leur grande différence puisqu'on passe (en valeur absolue) de 220 à 7 . Cette observation révèle une caractéristique importante de l'évolution diachronique des emplois de en matière (de) : quoique ce marqueur voie son usage comme TOP s'accroître de manière spectaculaire entre le XIX et le $\mathrm{XXI}^{\mathrm{e}}$ siècle (dans notre corpus : cf. diagramme 1 supra), cet essor concerne avant tout ses emplois intraprédicatifs, et non extraprédicatifs. A cet égard, la comparaison avec sur le plan (de) et dans le domaine (de) ne manque pas d'intérêt: nous avons souligné supra (commentaire du diagramme 4) que ces trois marqueurs se différencient de tous les autres en ceci que le contraste entre leur usage comme TOP au $\mathrm{XIX}^{\mathrm{e}}$ et au $\mathrm{XXI}^{\mathrm{e}}$ siècles apparaît comme très marqué (valeur absolue des indices de sur- et de sous spécificité $>200$ ). Or si ce contraste tend à s'évanouir (même s'il reste significatif) pour en matière de dans le diagramme 5 (du fait, répétons-le, de ses emplois de TOP essentiellement intraprédicatifs), tel n'est pas le cas des deux autres marqueurs : preuve que leur usage comme TOP extraprédicatif au $\mathrm{XXI}^{\mathrm{e}}$ siècle diffère aussi nettement de celui qu'il était au $\mathrm{XIX}^{\mathrm{e}}$ siècle.

On gagne enfin à étendre au marqueur au sujet (de) la comparaison des indices présentés dans les diagrammes 4 et 5 . Leur évolution se révèle en effet à la fois très proche et aux antipodes de celle de en matière (de). Proche, car l'on observe une chute tout aussi spectaculaire de la valeur absolue de l'indice des spécificités (=29) affecté aux emplois TOP extraprédicatifs de au sujet (de) (diagramme $5)$ alors qu'elle était très élevée $(=210)$ pour ses emplois TOP (intra- et extra-prédicatifs : cf. diagramme 4). Si l'emploi comme TOP de ce marqueur a donc significativement changé en un siècle, ce changement - comme pour en matière de - concerne moins ses emplois extraprédicatifs que ses emplois intraprédicatifs. Aux antipodes enfin, parce que l'évolution globale des emplois TOP de au sujet de est à rebours de celle de en matière de : alors que la première connaît un déclin général (plus ou moins creusé selon qu'on se focalise sur les seuls emplois extraprédicatifs ou bien sur tous), la seconde connaît au contraire un essor (limité aux TOP intraprédicatifs). 


\section{Conclusion}

Les données quantitatives présentées dans cette étude montrent que les emplois de sur le plan (de), au niveau (de), dans le domaine (de), dans le secteur (de) ont connu un essor remarquable dans Le Figaro entre la fin du $\mathrm{XIX}^{\mathrm{e}}$ et le début du $\mathrm{XXI}^{\mathrm{e}}$ siècle. Cet essor que l'on peut imputer au fait qu'elles ont commencé à se grammaticaliser comme des marqueurs de topique de discours contraste avec les données rassemblées sur quant à qui n'a fait, pendant le même temps, que se maintenir dans cette fonction où il est de très loin le plus usité. Au sujet de et à propos de qui avaient, comme quant $\grave{a}$, plusieurs siècles d'avance sur les expressions comme sur le plan (de), au niveau (de), dans le domaine (de), dans le secteur (de), paraissent par contre en perte de vitesse par rapport à quant à, surtout pour les emplois extraprédicatifs. Pour ce qui concerne enfin en matière (de), ses emplois comme Marqueur de Topique de Discours restent au début du $\mathrm{XXI}^{\mathrm{e}}$ siècle à un bon niveau, mais essentiellement comme intraprédicatifs.

Par ailleurs, si sur le plan (de), au niveau (de), dans le domaine (de), dans le secteur (de) ont connu un même essor de la fin du $\mathrm{XIX}^{\mathrm{e}}$ au début du XXI siècle, il demeure des différences importantes entre d'un côté sur le plan (de) et dans le domaine (de) et, de l'autre, dans le secteur (de) et au niveau (de). Ces différences demanderaient à être précisées et testées sur plus d'extraits que ceux que nous avons pu annoter, et sur d'autres expressions comme en regard de ou du point de vue (de) qui pourraient être ajoutées à celles que nous avons prises en compte dans cette étude. Il y a en effet tout lieu de penser que, dans le domaine qui nous préoccupe comme dans beaucoup d'autres, les marqueurs linguistiques assument des fonctions très spécifiques qui doivent être étudiées pour elles-mêmes, en synchronie, comme le font Delahaie \& Flament-Boistrancourt (2013) à l'oral pour niveau, par rapport à et en termes (de), et Flament-Boistancourt (2002) pour au niveau (de). On renvoie également à Velghe et Lahousse (2015) et Velghe (2015) qui apportent des données synchroniques sur, notamment, au niveau (de), au sujet de, en matière (de) en français parlé et écrit (corpus Le Monde) et le "Yahoo-based Constrastive Corpus of Questions and Answers » (entre l'écrit et l'oral).

Le travail ne manque donc pas sur le sujet, sans compter un aspect que nous n'avons pas discuté ici mais qui ressort nettement de la fréquentation de notre corpus : très souvent, les emplois comme marqueurs de topique de discours étudiés dans cet article apparaissent en série, sur des fragments de texte relativement étoffés.

\section{Références bibliographiques}

ANSCOMBRE J.-C. (2006), « Les locutions quant à, pour ce qui est de, en ce qui concerne. Chronique d'un discours annoncé », Modèles Linguistiques, 25.2, 155-169.

Charolles M. (1997), "L'encadrement du discours - Univers, Champs, Domaines et Espaces », Cahier de Recherche Linguistique, 6, 1-73.

ChOI-Jonin I. (2003), «Ordre syntaxique et ordre référentiel : Emplois de la locution prépositive quant $\grave{a} »$, in B. Combettes, C. Schnedecker \& A. Theissen (éds), Ordre et distinction dans la langue et le discours, Paris : Champion, 133-147.

Combettes B. et Prevost S. (2001), «Évolution des marqueurs de topicalisation », Cahiers de Praxématique 37, 103-124.

Combettes B. \& Prevost S. (2003), « Texte argumentatif et topicalisation d'une proposition : une approche diachronique », Scolia 16, p. 63-75

DebaisieuX J.-M. (2001), "Contraintes syntaxiques et discursives des emplois de quant à et en ce qui concerne dans les corpus oraux », Cahiers de praxématique 37, 125-164.

Delahaie J. \& Flament-Boistrancourt D. (2013), «Questions de variation : autour de quelques locutions méconnues de l'oral, niveau, par rapport à, en termes de. ", in Actes du colloque LPTS, 
"Across the line of speech and writing variation " (octobre 2011, Louvain-la-Neuve), Presses Universitaires de Louvain, collection « Corpora and Language in Use ».

Flament-Boistrancourt D. (2002), «Et au niveau tarifs, ça donne quoi ? L'éclairage du corpus Lancom sur le mot niveau », in J. Binon, P. Desmet, J. Elen, P. Mertens \& L. Sercu. (éds), Tableaux vivants. Opstellen over taal-en-onderwijs aangeboden aan Mark Debrock, Leuven : Universitaire Pers, 117-132.

FlØтtum K. (1999), «Quant à - thématiseur et focalisateur », in C. Guimier (éd.), La thématisation dans les langues. Berne : Peter Lang, 135-149.

FrASER B. (2009a), « Towards a theory of discourse markers », in K. Fischer (ed.) Approaches to Discourse Particles, Amsterdam: Elsevier Ltd, 189-204

FRASER B. (2009b), « Topic Orientation Markers », Journal of Pragmatics 41, 892-898

Goutsos D. (1996), « A model of sequential relations in expository text », Text 16 (4), 501-533.

GUIMIER C. (1996), Les adverbes du français. Le cas des adverbes en -ment, Paris : Ophrys.

KupPEVElt J. Van (1995), «Discourse Structure, Topicality and Questioning », Journal of Linguistics 31, 1, 109-147.

LAFON P. (1980), «Sur la variabilité de la fréquence des formes dans un corpus », Mots 1, 127-165.

Lagae V. (2003), "Quant aux livres/De livre, il n'en a lu aucun. Étude syntaxique de deux constructions détachées », Linguisticae Investigationes 26.2, 237-260.

Lagae V. (2011), "A propos de: un marqueur thématique très particulier », in D. Amiot, W. De Mulder, E. Moline \& D. Stosic (éds), Ars Grammatica. Hommage à Nelly Flaux, Berne : Peter Lang, 273-288.

LAMIROY B. \& CHAROLLES M. (2005), "Constitution et exploitation de corpus dédiés à l'analyse linguistique des adverbiaux ", in A.Condamines ed., Sémantique et corpus, Paris Hermès, 109-146

Lebart L. \& Salem A. (1994), Statistique Textuelle, Dunod : Paris.

MARANDIn J.-M. (1988), «À propos de la notion de thème de discours. Éléments d'analyse dans le récit », Langue française 78, 67-87.

Porhiel S. (1998), Les indicateurs d'intérêt, Thèse, Université de Paris 13/Presses du Septentrion.

PORHIEL S. (2001), "Au sujet de et à propos de - une analyse lexicographique, discursive et linguistique », Travaux de linguistique 2001/1, 42-43, 171-181.

Porhiel S. (2003), «Les indicateurs d'intérêt dans l'organisation textuelle », in B. Combettes, C. Schnedecker \& A. Theissen (éds), Ordre et distinction dans la langue et le discours, Paris : Champion, 425-442.

PORHIEL S. (2004), « Les introducteurs de cadre thématique », Cahiers de lexicologie 85, 2004-2, 945.

Prevost S. (2003), «Quant à : analyse pragmatique de l'évolution diachronique $\left(14^{\text {ème }}-16^{\text {ème }}\right.$ siècles) ", in B. Combettes, C. Schnedecker \& A. Theissen (éds), Ordre et distinction dans la langue et le discours, Paris : Champion, 443-459.

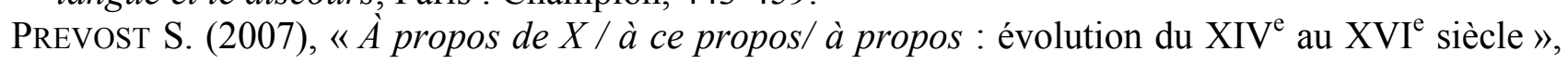
Langue française 156, 108-126.

Prevost S. (2008), «Quant à X et à propos de X du $14^{\text {ème }}$ au $16^{\text {ème }}$ siècle : émergence de deux marqueurs de topicalisation », L'information grammaticale 118, 38-43.

Prevost S. (2010), «Quant à X: du complément à l'introducteur de topique en passant par l'introducteur de cadre », in B. Combettes, C. Guillot, E. Oppermann-Marsaux, S. Prévost \& A. Rodríguez Somolinos (éds), Le changement en français. Etudes de linguistique diachronique, Bern : Peter Lang, 325-343.

PRÉvost S. (2011), «A propos : from verbal complement to 'utterance marker' of discourse shift », Linguistics $49: 2$, 391-413.

ReInhart T. (1980), « Condition for text coherence », Poetics to day 1/4, 161-180.

ReInhart T. (1981), «Pragmatics and linguistics: An analysis of sentence topics », Philosophica 27, 53-94.

RoBerts C. (2004), « Discourse context in dynamic interpretation », in L. Horn \& G. Ward (eds), Handbook of Contemporary Pragmatic Theory, Oxford: Blackwell, 197-220. 
Stein A. \& Schmid H. (1995), «Etiquetage morphologique de textes français avec un arbre de décisions ", Revue T.A.L Vol. 36.

TRAugOTT, E. \& E. KÖNIG (1991), « The semantics-pragmatics of grammaticalization revisited», in Traugott, E. \& Heine B. (eds.), Approaches to grammaticalization, Amsterdam: Benjamins, 189218.

Traugott, E. \& Dasher B. (2002), Regularity in Semantic Change, Cambridge : Cambridge University Press.

VAN DiJK T. A. (1977), "Sentence Topic and Discourse Topic », Papers in Slavic Philology 1, 1977, 49-61

Velghe T. \& LAHOUSSE K. (2015), «Thematic markers in informal written French : pour ce qui est de, au niveau (de), and en matière de », Journal of French Language Studies vol. 25 (3), 423444.

Velghe T. (2015), Les marqueurs de thématisation en français : Syntaxe, Discours, Prosodie, Thèse de Doctorat, KU Leuven. 